\title{
Editorial
}

\section{Prevention of Cervical Cancer}

According to WHO report in 2018 globally cervical cancer is the fourth most common cancer in women. An estimated 570,000 new cases were diagnosed in this year (2018) which represent $6.6 \%$ of all female cancers. The report also mentioned that approximately $90 \%$ of all deaths occurred in low and middle income countries. According to WHO report in $\mathbf{2 0 1 8}$ globally this high mortality rate from cervical cancer could be reduced through a comprehensive approach like effective vaccination program, screening and early diagnosis with treatment programmes. In Bangladesh each year 12,000 cases of cervical cancer are detected out of which $6600 \mathrm{die}^{1}$.

Cervical cancer belongs to a glorious natural history which favours prevention of this dreadful cancer. Fortunately its etiology also has be established. In 2018, the Nobel Prize in Physiology or Medicine was awarded to Dr. Haraldzur Hausen for his role in establishing the causal link between human papillomavirus (HPV) infection and cervical carcinoma. This announcement retlects the importance of the discovery of a sexually transmitted infection as the necessary cause of cervical cancer and the enormous opportunity for public health interventions ${ }^{2}$.

Among all cancers of the body cervical cancer belongs to a crystal clear and assuring natural history. The persistent infection of exocervical epithelium by HPV virus leads to the preneoplastic changes in the cervical epithelium. This change is known as cervical intraepithelial neoplasia (CIN) which is graded as CIN $\mathrm{I}, \mathrm{II}$ and III depending on degree of involvement of cervical epithelial thickness. CINI also known as low grade squamous intraepithelial lesion or LSIL is a transient HPV infection, remain static or regress spontaneously. Only $1-3 \%$ of $\mathrm{CINI}$ can progress to invasive cancer and it takes $5-25$ years. Ninety nine percent of $\mathrm{CINI}$ needs only observation. When treatment needed is usually by cryotherapy or thermocoagulation. CINII and III also known as high grade squamous intraepithelial lesion or HSIL. Around $30-50 \%$ of them progress to invasive cancer if they remain undetected and untreated. It also takes $5-15 \%$ years to progress to invasive cancer. CIN II and III are known as cervical cancer precarsors. This ensuring natural history and well established eitiology of cervical cancer is the background of prevention of this cancer. Treatment of CINIII is by Loop Electrosurgical Excision Procedure (LEEP) or Cold Knife Conisation (CKC). CINII is a ill defined condition, treatment of which is not well established. Most of this lesions can be treated by ablative procedure and very few need excisional procedure. Very recently facility for determing E6 oncoprotein has been developed at BSMMU depending on it's presence or absence on cervical sample, decision for treatment or observation can be undertaken. Presence of E6 oncoprotein in cervical sample of CINII cases need treatment. On the other hand absence of which do not need treatment.

Carcinoma-in-situ cervix (CIS) is a different category of disease characterized by disordered growth of whole thickness of cervical epithelium together with malignant changes of some cells with intact basement membrane. Treatment of this condition is also different, either by CKC or by LASER cone biopsy. Most of these cases undergo changes to invasive cancer if not detected and treated earlier.

Key strategies for prevention of Cervical Cancer: All women can avail the opportunity for prevention of cervical cancer at 3 phases of her life as shown in the figure-1.

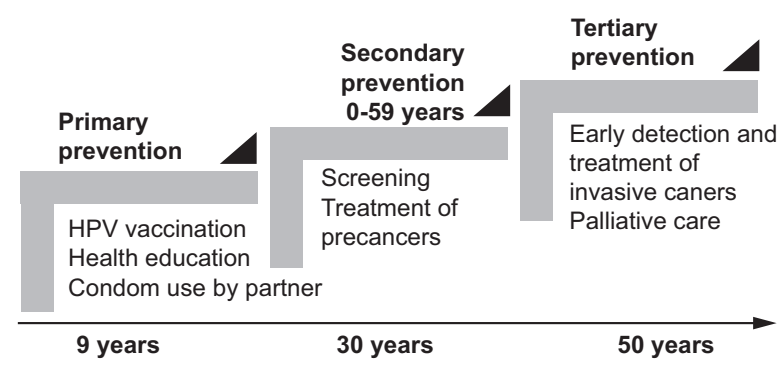

Fig.-1: Cervical cancer prevention and control strategies over the lifespan of women ${ }^{3}$

Primary prevention ${ }^{4}$ : The HPV vaccine, which received FDA approval in 2005 in over $90 \%$ effective 
when given to sexually unexposed girls of 9 to 15 years old. This group of girls require 2 (two) doses only at 0 and 6 months. And they are considered as target group. Second group of women are of 16 to 26 years old women. They can receive the vaccine and considered as catch up group. They need 3 doses of vaccine at 0,1 and 6 months. HPV vaccines are highly recommended for both girls and boys. Boys can get the vaccine at around age 11 to 12 to prevent them from developing HPV related cancers including anal, rectal, mouth / throat and penile cancers.

Australia was the first country in the world to initiate a national publicly funded HPV vaccination programme and to document it's effects on intermediate outcomes. Australia enrolled national HPV vaccination programme in 2007, used a quadrivalant vaccine ( Gardasil [Merck]) in a three dose schedule ${ }^{5}$. Boys aged 12 to 13 years were included in the programme from $2013^{6}$. In UK national immunization programme is using HPV vaccine. The programme uses Gardasil and the girls are offered the childhood immunization programme in which the vaccine is routinely given to 12 to 13 years girls with 2 doses at 0,6 months. But it is available free on National Health Service (NHS ) to all girls up to 18th birthday ${ }^{7}$.

In Bangladesh a demonstration project has been started at Gazipur district during 2016 - 2017 . This programme included all girls at their 10 th birth year or all school girls of class V. In this programme 33000 girls of Gazipur district have been vaccinated by two doses of bivalent vaccine (Cervarix [GSK]). Recently an evaluation programme is going on to see the feasibility of scaling up of the programme to incorporate HPV vaccine in national immunization programme of Bangladesh (personal communication with Dr. Habibullah Talukder). Some risk factors for development of cervical cancer can be avoided by widespread health education on avoidance of childhood marriage, repeated childbirth, multiple sexual partner, smoking, use of oral contraceptive pill for more than 5 years needs to be ensured. Health education and vaccination can prevent $70 \%$ of cervical cancer.

Secondary Prevention: Secondary prevention of cervical cancer by screening is the oldest model of global and national health policy. Secondary prevention is feasible through the detection and timely treatment of HSIL and carcinoma in-situ.
In UK cervical cancer mortality have been reduced by $70 \%$ through the introduction of the NHS cervical screening programme in 1988. In England a population based organized screening programme is actively maintaining call and recall system for screening by cytology. Recent research concluded that the risk of cervical cancer can be reduced to $70 \%$ if everyone attended when invited. In this programme cytology based screening is offered to all women aged $25-64$ at the interval of 2 years ${ }^{8}$. In Australia the incidence and mortality of cervical cancer are among the lowest in the world. After introduction of the National Cervical Screening Programme (NCSP) in 1991, cervical cancer incidence in Australia decreased by approximately $50 \%$ in women elder than 25 years $^{9}$. The NCSP involved cyclogy-based screening every 2 years from age 18-20 years to age 69 years. Coverage of this programme is reported in 2017 as $83 \%$ over the 5years period $2011-15^{10}$.

Situation of South East Asian Region is different. The public health system of this region is overburdened and has limited copability to include additional health programme. According to Indian Health Service cytology-based screening every three years is recommended for all women aged 21 to 65 years and combined screening by paps and HPV test recommended every five years for women aged 3065 years $^{11}$. In Bangladesh a National Breast and Cervical Screening Programme is going on since 2015. In this programme opportunistic VIA screening is offered by trained medical personnel to healthy women aged 30 to 59 years attending health care facilities in 44 districts. Colposcopy and/or directed biopsies are performed on VIA-positive women at Bangabandhu Sheikh Mujib Medical University (BSMMU) or medical college hospitals. From January 2005 to June 2008, 104098 women underwent VIA screening. Among them 5013 (4.8\%) women were positive, of whom 4371 (87.2\%) attended colposcopy clinics in different hospitals. Results for sensitivity, specificity, and positive predictive value of VIA to detect CIN 2-3 lesions were 93.6\%, 58.3\%, and $15.6 \%$, respectively ${ }^{12}$.

Cytology based screening in Bangladesh has been developed tremendously during last 5 years. Almost all medical college and some district hospitals has developed pap's smear facility. In BSMMU Liquidbased Cytology (LBC) has been introduced in 2015, 
and now it is in routine use. One study report at BSMMU shows its specificity as $92 \%$. Though sample collection is easy by LBC sample, it cannot be introduced everywhere as the method is technically difficult and costly.

\section{Prof. Sabera Khatun}

MBBS, FCPS, FICS, Fellow Gynaecologic Oncology

Chairman

Dept. of Gynaecological Oncology

Bangabandhu Sheikh Mujib Medical University

Shahbag-1000

References:

1. World Health organization; https://int/prevention/ diagnosis-screening/cervical-cancer/en/

2. Alexanda de Pokomandy, MDCM, MSc, MarrieHelene Mayrand, MD,Phd. HPV Infection Epidemiology and Prevention, Ed- Ali Ayhan, Nicholas Reed, Murat Gultekin, Polat Dursun, Text book of Gynaecological Oncology $4^{\mathrm{TH}}$ edition; ESGO (European Society of Gynaecological Oncology) ;2017, p-195.

3. World Health organization;Strategic framework for the Comprehensive Control of Cancer Cervix in South-East Asia Region. 2015,p-5.

4. UPMC health beat; https://share.upmc.com/ 2018/02/cervical-cancer-health-crisis/

5. Department of Health and Ageing. Immunise Australia program: human papillomavirus (HPV). February14, 2013.http:// www.health.gov.au/internet/immunise/ publishing.nsf/Content/immunise-hpv(accessed May 9, 2013).

6. Li N, Franceschi S, Howell-Jones R, Snijders PJ, Clifford GM. Human papillomavirus type distribution in 30,848 invasive cervical cancers worldwide: variation by geographical region, histological type and year of publication. Int $\mathrm{J}$ Cancer 2011; 128: 927-35.

7. Cervical cancer-Prevention-NHS; Prevention Cervical Cancer; https://www.nhs.uk/conditions/ cervical-cancer/prevention/

8. Stubbs R. Cervical Cancer Prevention week 2018 -PHE Screening; 26 January 2018-NHS Cervical Screening Programme; https:// phescreening .blog.gov.uk/201801/26/cervicalcancer-prevention-week-2018/

9. Smith M, Canfell K. Impact of the Australian National Cervical Screening Program in women of different ages. Med J Aust 2016; 205: 359-64.

10. Australian Institute of Health and Welfare. Cervical screening in Australia 2014-2015. May 25, 2017. https://www.aihw.gov.au/reports/ cancer-screening/cervical-screening-inaustralia-2014-2015/contents/ table-of-contents (accessed March 21, 2018).

11. Andria Apostolou. Cervical Cancer Awareness and Prevention, Indian Health Service; The Federal Health Program for American Indians and Alaska Natives, January 22,2018; https:www.ihs.gov/newsroom/his-blog/ jauary2018/cervical-cancer-awarencess-andprevention/

12. Nessa A, Hussain MA, Rahman JN, Sankaranarayanan R. Screening for cervical neoplasia in Bangladesh using visual inspection with acetic acid; International journal of gynaecology and obstetrics: 2010;111(2): 115-8. 